Revista de Matemática: Teoría y Aplicaciones 2013 20(1) : 21-34

CIMPA - UCR ISSN: $1409-2433$

\author{
SIMULATION OF DECAMETRIC WAVE \\ PROPAGATION IN THE IONOSPHERIC PLASMA
}

\title{
SIMULACIÓN DE PROPAGACIÓN DECAMÉTRICA DE ONDAS EN EL PLASMA IONOSFÉRICO
}

\author{
KSENIYA S. Kiryanova* AndRey S. KRyUKOVSKY ${ }^{\dagger}$ \\ DMITRIY S. LUKIN ${ }^{\ddagger}$
}

Received: 31/Dec/2011; Revised: 10/May/2012; Accepted:

$$
\text { 27/Nov/2012 }
$$

*Postgraduate student, Russian New University, 22, Radio Street, Moscow, 105005, Russia. E-Mail: 2661348@mail.ru

${ }^{\dagger}$ Head Chair of Information Technologies and Natural Sciences, Russian New University, Moscow, Russia. E-Mail: kryukovsky@rambler.ru

${ }^{\ddagger}$ Head Chair of Physics and Mathematical Problems of Wave Processes, Moscow Physical and Technical Institute (State University), 9, Institutskiy pereulok, Dolgoprudniy, Moscow region, 141700, Russia. E-Mail: lukin@mail.mipt.ru 


\begin{abstract}
The decametric radiowaves propagation in the ionospheric plasma was considered. Bicharacteristic system of equations was used in the modeling ray structures. This system, which allows finding the ray paths with great precision, was obtained by D.S. Lukin. Models of the ionosphere with horizontal gradients were used in the calculations; local inhomogeneities, such as reduced electron density in the maximum layer and increased electron density below the maximum layer, were taken into account. The features of shortwave propagation, such as getting a wave of interlayer channel, distribution and out of it, reflection of waves from different ionospheric layers were explored. Was shown that the rays can be ejected from the field of distribution and curled when hit in the inhomogeneity of the ionosphere. The formation of caustic surfaces and its dynamics were studied. The radiowaves propagation in the Earth's ionosphere was studied. This ionosphere profiles were restored the scientific group led by Professor V.E. Kunitsyn by radiotomography method based on real observations. Ray structures in the area of "equatorial anomaly" were built. The models, both including and excluding the effect of Earth's magnetic field and the influence of the equatorial anomaly were considered. Ray structures at the different positions of the radiation source, as well as different operating frequency, were investigated. Dynamic model of the ray's propagation was developed.
\end{abstract}

Keywords: Decameter radiowaves, the bicharacteristic system, ionospheric plasma, the equatorial anomaly, ray structures.

\title{
Resumen
}

Se considera la propagación de radioondas decamétricas en el plasma ionosférico. Se usa un sistema bicaracterístico de ecuaciones para modelar las estructuras radiales. Este sistema, que permite encontrar los caminos radiales con gran precisión, fueobtenido por D.S. Lukin. En los cálculos se usaron modelos de la ionosfera con gradientes horizontales; se tomaron en cuenta inhomogeneidades locales, tales como densidad reducida de electrones en la capa máxima y dendidad aumentada de electrones bajo la capa máxima. Se exploraron características de la propagación de las ondas cortas. Se muestra que los rayos pueden ser expulsados del campo de distribución y hacer un bucle cuando chocan con la inhomogeneidad dela ionosfera. Se estudia la formación de superficies cáusticas y su dinámica. También se estudia la propagación de radioondas en la ionosfera de la Tierra. Los perfiles de esta ionosfera fueron dados al grupo liderado por el Profesor V.E. Kunitsyn por medio del método de radiotomografía basado en observaciones reales. Se construyeron estructuras de rayos en el área de "anomalía ecuatorial". Se consideraron que incluyen y que excluyen el efecto del campo magnético de la Tierra y la influencia de la anomalía ecuatorial. Se investigaron estructuras radiales 
en diferentes posiciones de la fuenta de la radiación, así como diferentes frecuencias operativas. Se desarrolló el modelo dinámico de la propagación de los rayos.

Palabras clave: Radioondas decamétricas, el sistema bicaracterístico, plasma ionosférico, anomalía ecuatorial, estructuras radiales.

Mathematics Subject Classification: 78A40.

\section{Ray method and ray paths}

There is always the need to solve various problems of distant radio communication, radio-navigation, radiolocation, over-the-horizon radio-sounding, as well as to study the structure of Earth's upper atmosphere - the ionosphere. Since the beginning of last century, decameter range electromagnetic waves are widely used for radio communications over long distances. As a consequence, the problem of studying the propagation of radio waves that range in the Earth's atmosphere is actually.

It is very important to choose the most efficient computational algorithms and schemes of calculating the propagation characteristics of radio signals in practice of radio-communication. Most common in virtue of its simplicity and clarity, have methods for calculating the decametric radio bi-characteristics developed by D. S. Lukin in the 70s of last century, based on the of geometric optic approximation. Thus, ray methods are traditional in the study of the propagation of short waves in various areas. Recently continued intensive studies of the propagation of radio waves in the Earth's ionosphere at precisely the method of bi-characteristics ([1], $[2],[3],[4])$.

In the modeling of ray trajectories the Hamiltonian bi-characteristic system of equations in an inhomogeneous medium, obtained by D. S. Lukin, in the form [5] had been used:

$$
\frac{d \mathbf{k}}{d t}=\frac{\partial \omega^{2} \varepsilon}{\partial \mathbf{r}}\left(\frac{\partial \omega^{2} \varepsilon}{\partial \omega}\right)^{-1} \quad \frac{d \mathbf{r}}{d t}=\left(2 c^{2} \mathbf{k}-\frac{\partial \omega^{2} \varepsilon}{\partial \mathbf{k}}\right)\left(\frac{\partial \omega^{2} \varepsilon}{\partial \omega}\right)^{-1}
$$

where $\mathbf{k}$ - the wave vector, $t$ - the parameter along ray trajectory, $\omega$ - the angular frequency of radiation, $\varepsilon$ - the effective permittivity of a propagation medium, $r$ - coordinates of the observation point, $c$ - light velocity, and

$$
\mathbf{k}=\left(k_{x}, k_{y}, k_{z}\right), \quad \varepsilon \equiv \varepsilon(\mathbf{r}, \mathbf{k}, \omega), \quad \mathbf{r}=(x, y, z) .
$$

Ray propagation is investigated in the case of the following models of the effective permittivity of the medium: a model of an inhomogeneous 
isotropic ionosphere (2) and the model of an inhomogeneous anisotropic ionosphere (3) [6].

$$
\begin{gathered}
\varepsilon=1-v, \\
\varepsilon_{ \pm}=1-\frac{2 v(1-v)}{2(1-v)-u \sin ^{2} \alpha \pm \sqrt{u^{2} \sin ^{4} \alpha+4 u(1-v)^{2} \cos ^{2} \alpha}},
\end{gathered}
$$

where sign "+" corresponds to the ordinary wave and the sign "-" corresponds to the extraordinary wave.

In 2, 3 the following notations are used:

$$
v=\frac{\omega_{p}^{2}}{\omega^{2}}=\frac{4 \pi e^{2} N}{m \omega^{2}} \quad u=\frac{\omega_{H}^{2}}{\omega^{2}}=\frac{e^{2} H_{0}^{2}}{m^{2} c^{2} \omega^{2}},
$$

where $e$-electron charge, $N$-electron density profile, $m$-mass of the electron, $H_{0}$ - the value of the magnetic field of the Earth, $\alpha$-the angle between the magnetic field of the Earth and the wave vector.

The expression for the cosine of the angle $\alpha$ is given by:

$$
\cos \alpha=\frac{H_{0 x} k_{x}+H_{0 y} k_{y}+H_{0 z} k_{z}}{H_{0}|\mathbf{k}|} .
$$

Models used take into account the frequency dispersion of the propagation medium. It is assumed that the radiation source is a point and located at coordinates:

$$
\left.\mathbf{r}\right|_{t=0}=\left(x_{0}, y_{0}, z_{0}\right),
$$

and the initial wave vector $k(0)$ parametrically depends on the ray angles:

$$
\begin{aligned}
& k_{x}(0)=\frac{\omega}{c} \cos \zeta \cos \eta, \\
& k_{y}(0)=\frac{\omega}{c} \sin \zeta \cos \eta, \\
& k_{z}(0)=\frac{\omega}{c} \sin \eta .
\end{aligned}
$$

In those calculations, which take into account the magnetic effect of the Earth, the amplitude of the magnetic field is assumed to be constant, and the orientation of the magnetic field relative to the local coordinate system is defined by two angles $\gamma$ and $\varphi$ :

$$
\begin{aligned}
& H_{0 x}=H_{0} \cos \gamma \cos \varphi, \\
& H_{0 y}=H_{0} \cos \gamma \sin \varphi, \\
& H_{0 z}=H_{0} \sin \gamma .
\end{aligned}
$$




\section{Models of the ionosphere}

We consider the propagation of radio waves in an anisotropic medium with the influence of Earth's magnetic field. The effective permittivity in this case is calculated by the formula (3). Consequently, there is a propagation of ordinary and extraordinary waves. Initially, propagation is carried out in the plane $(x, z)$. The radiation source located at the origin $\left(x_{0}=y_{0}=z_{0}=0\right)$. A model of two-layer ionospheric plasma, for which the electron density profile has the form [1]:

$$
\begin{aligned}
N(\mathbf{r})=N_{0}\{ & \exp \frac{1}{2}\left[1-\left(\frac{z-z_{01}}{z_{m 1} / 2}\right)\right. \\
& \left.-\sec \chi \exp \left(-\left(\frac{z-z_{01}}{z_{m 1} / 2}\right)\right)\right] \\
& \left.+\beta \exp \left[-\left(\frac{z-z_{02}}{z_{m 2}}\right)^{2}\right]\right\},
\end{aligned}
$$

where $N_{0}$ - the electron density at the peak of the main layer $F 2, z_{01}$ height of the maximum layer $F 2, z_{m 1}$-effective half-layer $F 2, \beta-$ a dimensionless coefficient characterizing the degree of ionization of the lower layer to the core, $z_{02}$-maximum height of the lower layer $E, z_{m 2}$-effective half-bottom layer $E$.

In Figure 1 the ray structure in the case of propagation of the ordinary wave in the plane $(x, z)$ with $\beta=0$ (one-layer model) is shown. It is evident that some rays penetrate through the ionospheric plasma, and the rest returned to the ground, forming a caustic edge. As a result there is the dead zone, which extends about $180 \mathrm{~km}$.

In contrast to the ordinary wave the ray structure of the extraordinary wave with the same parameters are fully reflected rays from the ionosphere.

In Figure 2 the extraordinary wave propagation in the case where the coefficient $\beta$ is equal to 0.55 (double-layer model) is shown. In addition, Figure 2 shows the double-jump wave propagation. It is clearly seen that the reflection comes from the two layers at once: at $100 \mathrm{~km}$ from the lower layer and at an altitude of $300 \mathrm{~km}$ from the base layer.

With decreasing values of $\beta$ only fewer rays will be reflected from the lower layer, the bulk of the rays will pass through the bottom layer. However, if the coefficient $\beta$ increase, the less rays will leak above the layer $E$. It is seen that after the dead zone, a region of unstable radio reception are arise, characterized by fading due to the interference of rays reflected from the layer as $E$, and $F 2$. 


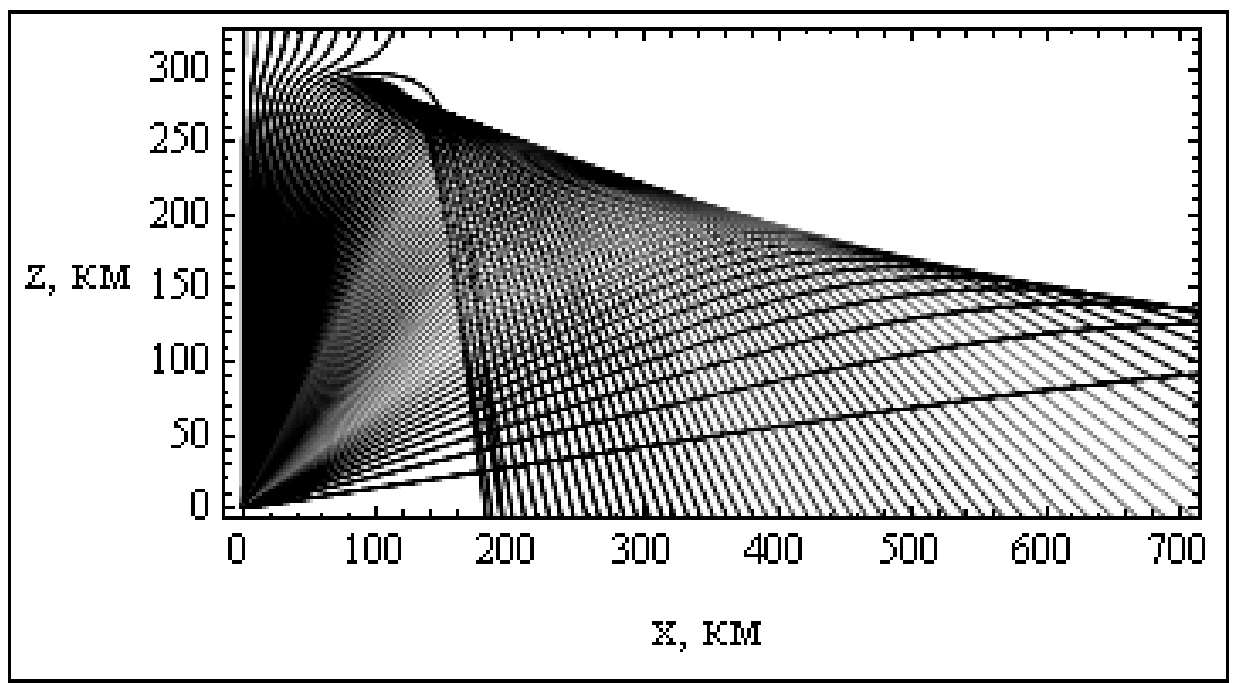

Figure 1: Ray structure in the plane $(x, z)$. One-jump propagation. The ordinary wave, $\gamma=\pi / 4$; carrier frequency of $12.8 \mathrm{MHz}$.

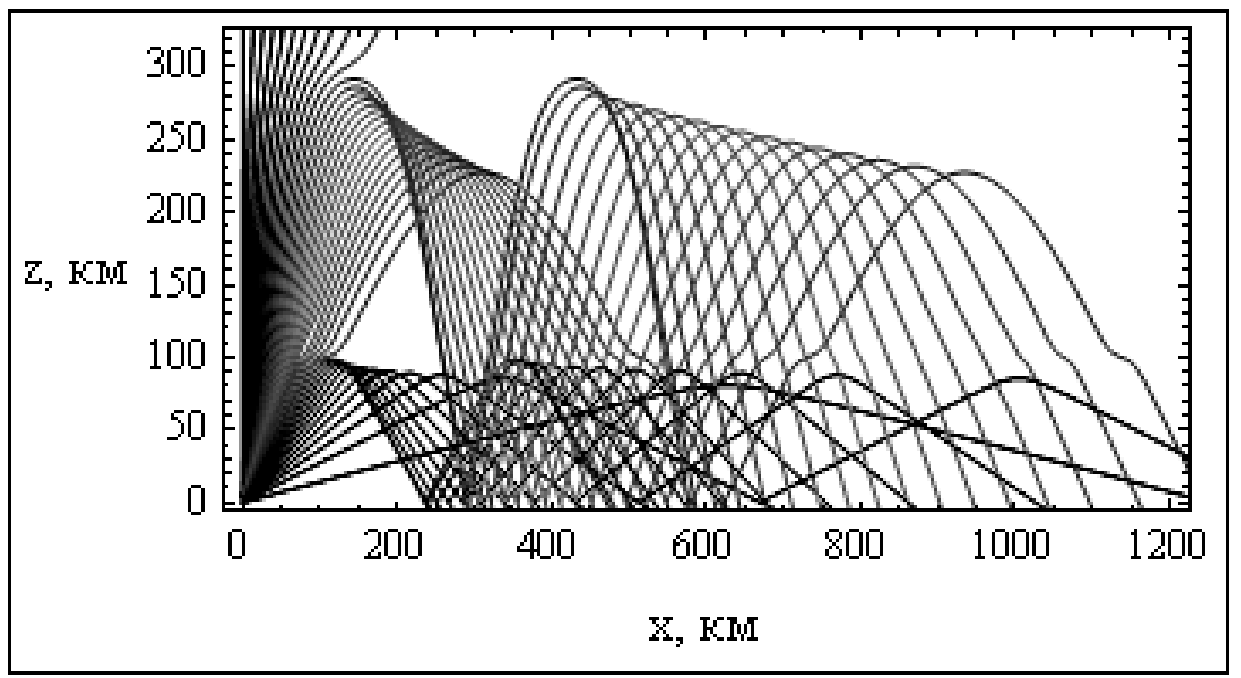

Figure 2: Ray structure in the plane $(x, z)$. Double-jump propagation. The extraordinary wave, $\gamma=3 \pi / 4$; carrier frequency of $13.5 \mathrm{MHz}$.

Often there is such heterogeneity as interlayer ionospheric channel. Figure 3 shows the spread of the extraordinary wave in the case of interlayer channel. It is clearly seen that the rays penetrate into the inter-layer channel and continues to propagate in it. You can see that radio reception is not possible rays, because the rays reflected from the $F 2$ layer does not 
return to Earth, and the signal came from a layer of $E$, may be too weak.

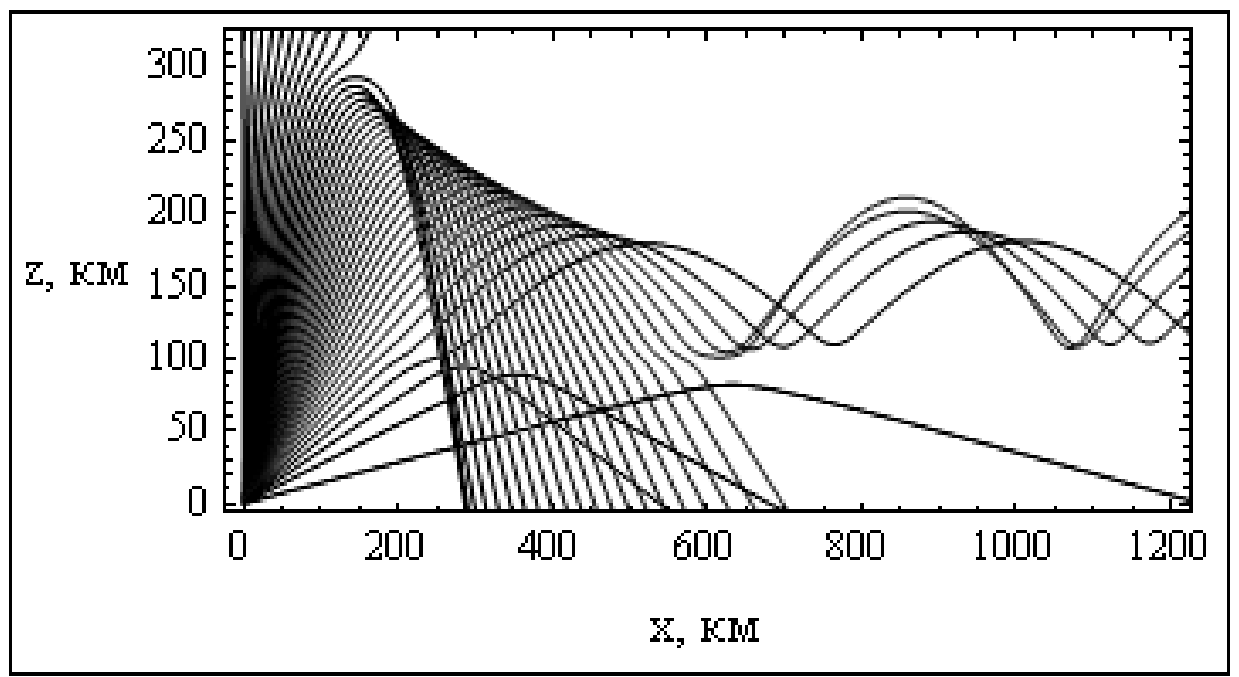

Figure 3: Ray structure in the plane $(x, z)$. The extraordinary wave $\gamma=3 \pi / 4$, $\beta=0.4$; the carrier frequency of $13.5 \mathrm{MHz}$.

The appearance of interlayer channel is possible by the following profile of the electron density [1]:

$$
\begin{aligned}
N(\mathbf{r})=N_{0}\{ & \exp \frac{1}{2}\left[1-\left(\frac{z-z_{01}}{z_{m 1} / 2}\right)\right. \\
& \left.-\sec \chi \exp \left(-\left(\frac{z-z_{01}}{z_{m 1} / 2}\right)\right)\right] \\
& \left.+f(x) \exp \left[-\left(\frac{z-z_{02}}{z_{m 2}}\right)^{2}\right]\right\},
\end{aligned}
$$

Here

$$
f(x)=\beta \frac{x}{x_{m}} .
$$

The case where the interlayer channel first appears and then disappears, we can be modeling by the function:

$$
f(x)=\beta \sin \left(\frac{\pi}{2} \frac{x}{x_{m}}\right) .
$$

Unlike the previous case, rays penetrate the interlayer channel, and then come out of it. 
Since the local inhomogeneities of the ionosphere of both natural and artificial origin have a significant influence on radio-wave propagation, this paper presents the modeling of such situations. In this case, the dependence of the electron density on the coordinates can be represented as $[1]$ :

$$
\begin{aligned}
N(\mathbf{r})=N_{0}\{ & \exp \frac{1}{2}\left[1-\left(\frac{z-z_{01}}{z_{m 1} / 2}\right)\right. \\
& \left.\left.-\sec \chi \exp \left(-\left(\frac{z-z_{01}}{z_{m 1} / 2}\right)\right)\right]+F(x, y, z)\right\} .
\end{aligned}
$$

Here

$$
\begin{aligned}
F(x, y, z)=\beta_{l o c} \exp [- & \left(\frac{x-x_{l o c}}{x_{m 3}}\right)^{2} \\
+ & \left(\frac{y-y_{l o c}}{y_{m 3}}\right)^{2} \\
& \left.\left.+\left(\frac{z-z_{l o c}}{z_{m 3}}\right)^{2}\right)\right],
\end{aligned}
$$

where $x_{m 3}, y_{m 3}, z_{m 3}$-inhomogeneity sizes, $x_{l o c}, y_{l o c}, z_{l o c}$-coordinates of the inhomogeneity location. In the modeling the size of the small inhomogeneity were taken:

$$
x_{m 3}=y_{m 3}=z_{m 3}=20 \mathrm{~km} .
$$

Let's see how will look the same radial structure in the event of a local inhomogeneity. If the region of high electron density lies outside the layer, as clearly seen in 4, this inhomogeneity pushes the rays from the propagation area and regions of the Earth with a low electromagnetic field intensity are appear.

If the region of low electron density lies at an altitude layer maximum, the ray bends around itself several times, as is clearly seen in Figure $5^{1}$. They also goes out of the propagation region, and rotates in the plane $(x, y)$ (Figure 6).

\section{The real ionosphere}

Let us consider the ray propagation in the case where the electron density profiles are constructed in the form of interpolation functions on the data

\footnotetext{
${ }^{1}$ V. I. Stasevitch had noted this opportunity for the first time.
} 


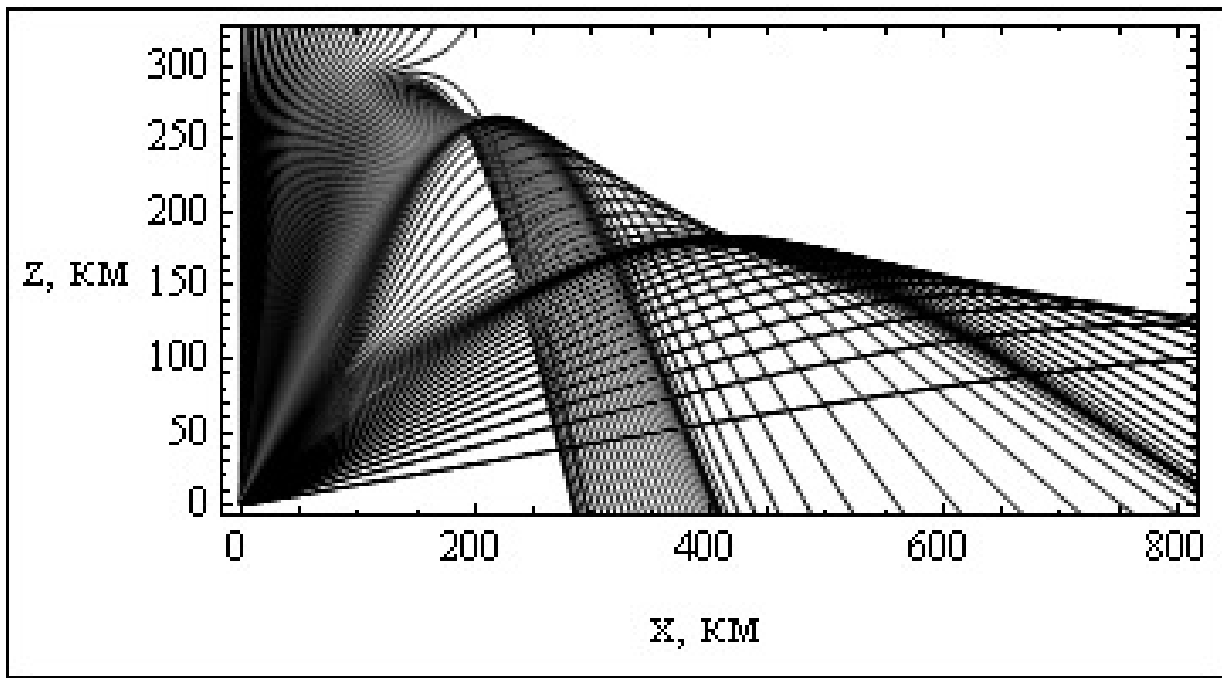

Figure 4: Ray structure in the plane $(x, z)$ in the presence of the local inhomogeneity. The extraordinary wave, $\gamma=3 \pi / 4$; the carrier frequency of $13.5 \mathrm{MHz}$.

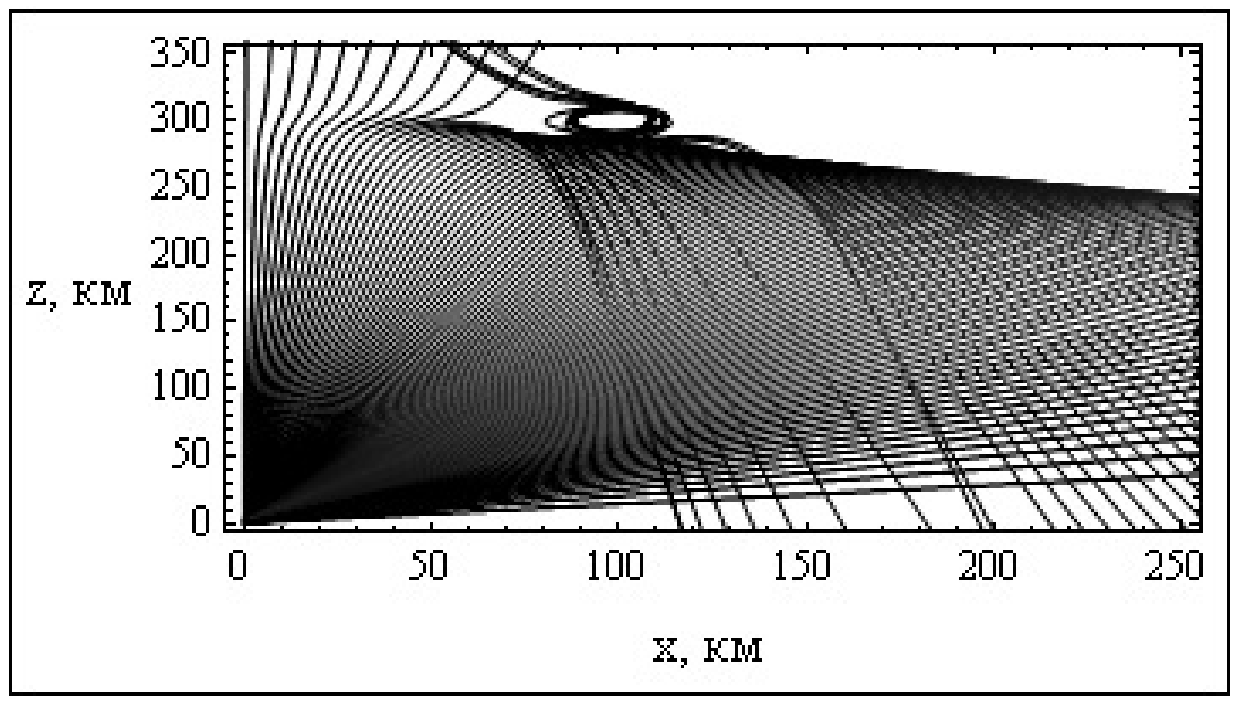

Figure 5: Ray structure in the plane $(x, z)$ in the presence of local inhomogeneity. The extraordinary wave, $\gamma=3 \pi / 4$; the carrier frequency of $13.5 \mathrm{MHz}$.

obtained in [7], [8], [9], [10], by the radio tomography. Reconstruction of layers $E$ and $F$ in the area of the equatorial anomaly along the meridian, corresponding to $121^{\circ}$ east longitude is built. The modeling results for the 


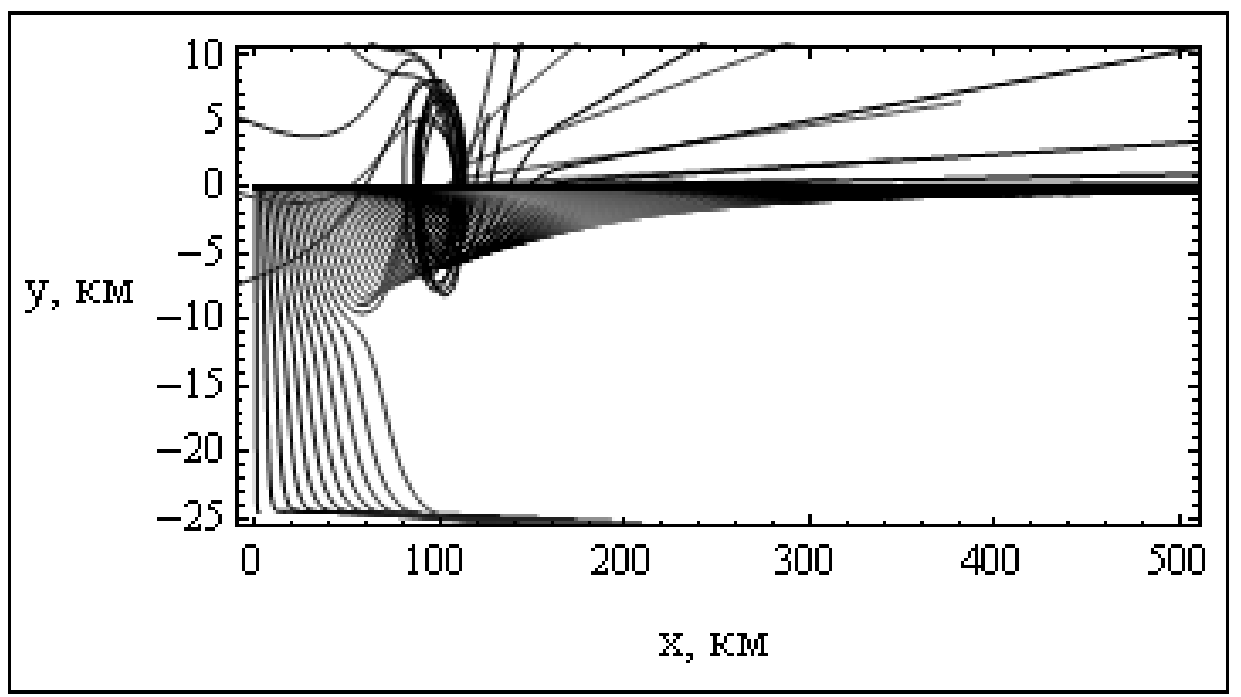

Figure 6: Ray structure in the plane $(x, y)$ in the presence of local inhomogeneity. The extraordinary wave, $\gamma=3 \pi / 4$; the carrier frequency of $13.5 \mathrm{MHz}$.

propagation of an isotropic media are presented. In this case, the effective permittivity is calculated by the formula (2).

Figure 7 illustrates the wave propagation in the case when the radiation source located at the Earth's surface at latitude of $20^{\circ}$ and emits a signal with a frequency of $12 \mathrm{MHz}$. It is seen that the core equatorial anomaly prevents the spread of rays and reflects them to the left. Part rays seeping through the ionosphere, but most of them reflected from the layer $E$.

Although the rays are reflected from the layer and $E$, and the layer $F$, the rays still pass through the ionospheric layer, which leads to the formation of so-called "dead zones" within which the signal reception is impossible. Note that the ray structure varies with the displacement of the radiation source. When the radiation source is located in the immediate vicinity of the equatorial anomaly, all the rays are reflected and can not propagate over large distances without reflections. It is clear that the equatorial anomaly can be a substantial obstacle in the path of propagation of electromagnetic waves.

When the radiation source is located above the layer peak electron density, all the rays go up, and on Earth radio reception impossible. Attention is drawn to the fact that the core of the equatorial anomaly shifts slightly over time, but also changes the maximum electron density. Thus, to ensure a high quality radio communication must take into account changes in the ionospheric plasma over time, and the impact of these changes on the radiowaves. 


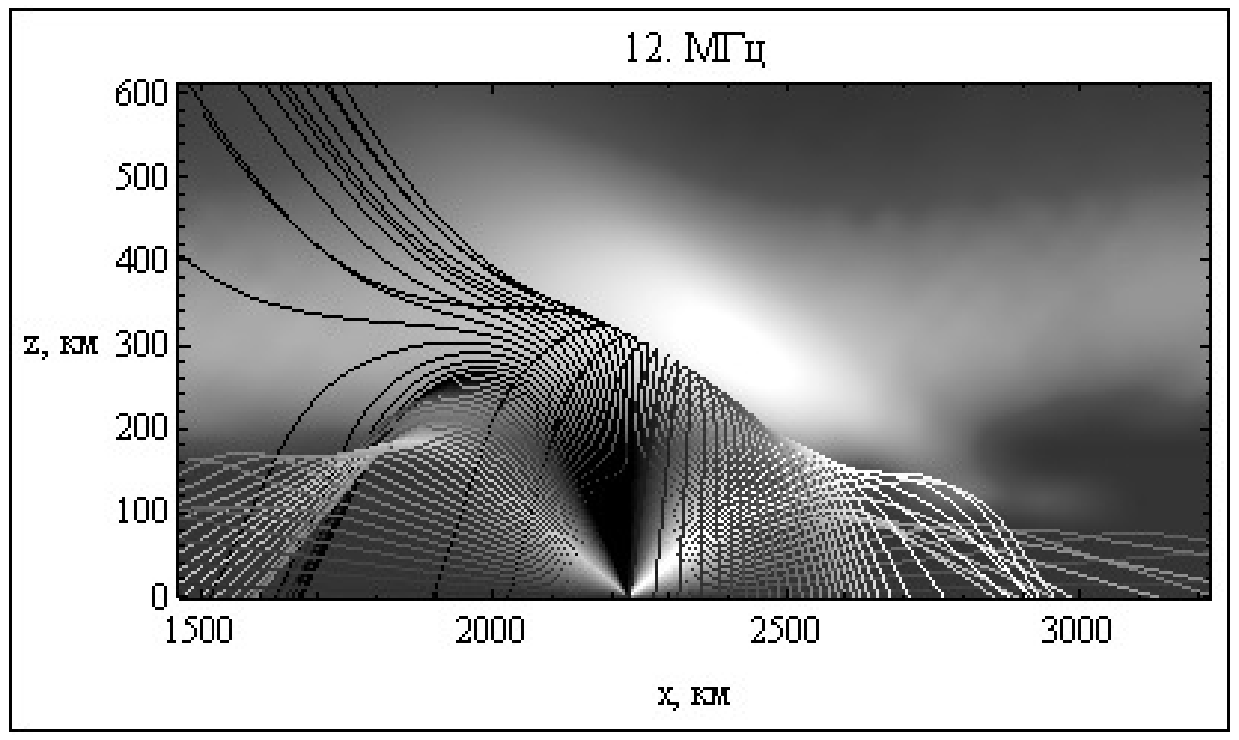

Figure 7: Ray structure. The radiation source is located on the surface of the Earth, the latitude of $20^{\circ}$

Consider the changing nature of signal propagation in the vicinity of the equatorial anomaly, caused by the radiation frequency changing. Figure 8 shows the ray structure in the case where the radiation source located at the same latitude of $20^{\circ}$ on the surface of the Earth, and the carrier frequency is $9 \mathrm{MHz}$. It is seen that the wave is totally reflected from the ionospheric plasma, the reflection height is about $240 \mathrm{~km}$. Note, however, that radio-waves propagate at more than $1000 \mathrm{~km}$. Figure 9 shows the change of the ray structure in case of increasing the carrier frequency of the radiation source. In this case, the radiation frequency is $12.5 \mathrm{MHz}$. The height of the reflection of rays increased. Now it is about $300 \mathrm{~km}$. Not all the rays are reflected from the ionosphere, and a "dead zone" is formed. It is clearly seen that the rays on the left goes up, the equatorial anomaly to the right reflects the rays. You may notice that the rays are reflected from the layer as $E$, and on the layer $F$. Upon reflection from the $E$ layer is formed caustic cusps, both right and left.

\section{Conclusion}

Thus, in the paper numerical modeling of short-wave signals was carried out. The propagation of decameter radio-waves with different models of the ionosphere: one-layer and double-layer models, one-jump and double-jump propagation, capturing the wave of inter-layer channel was investigated. 


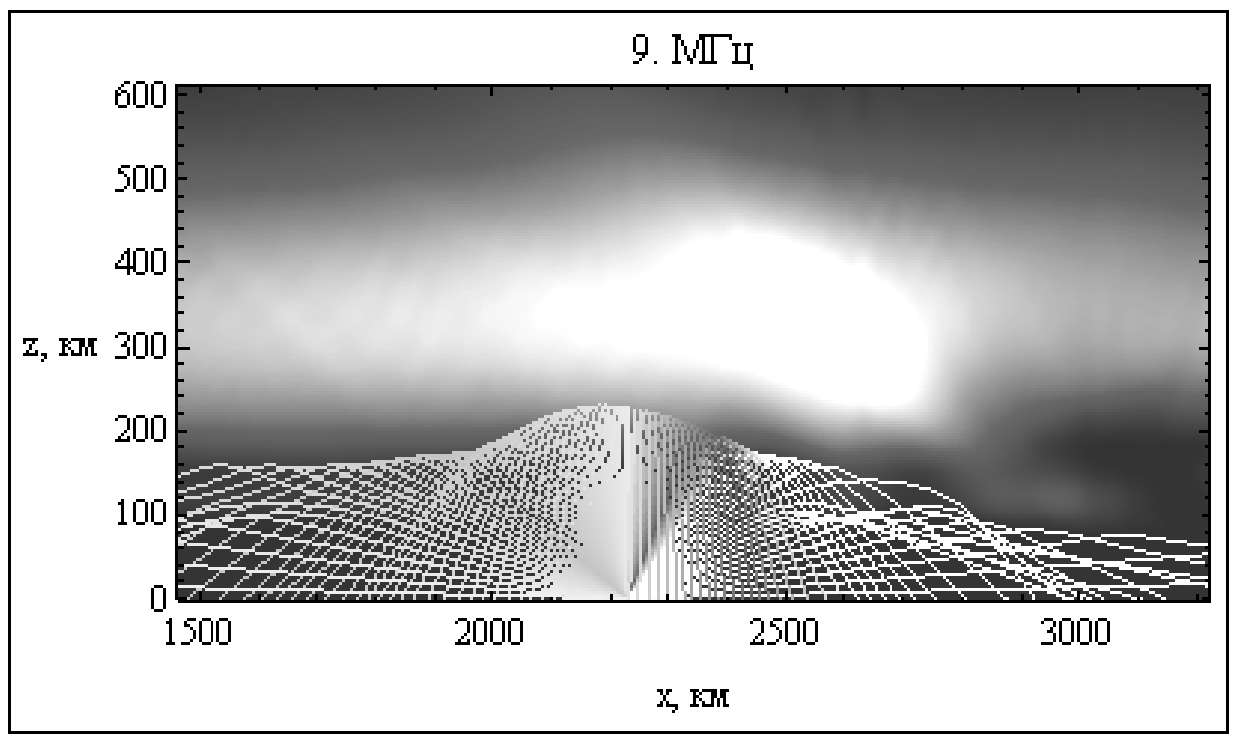

Figure 8: Ray structure. The source of radiation on the Earth, the latitude of $20^{\circ}$ the frequency of $9 \mathrm{MHz}$.

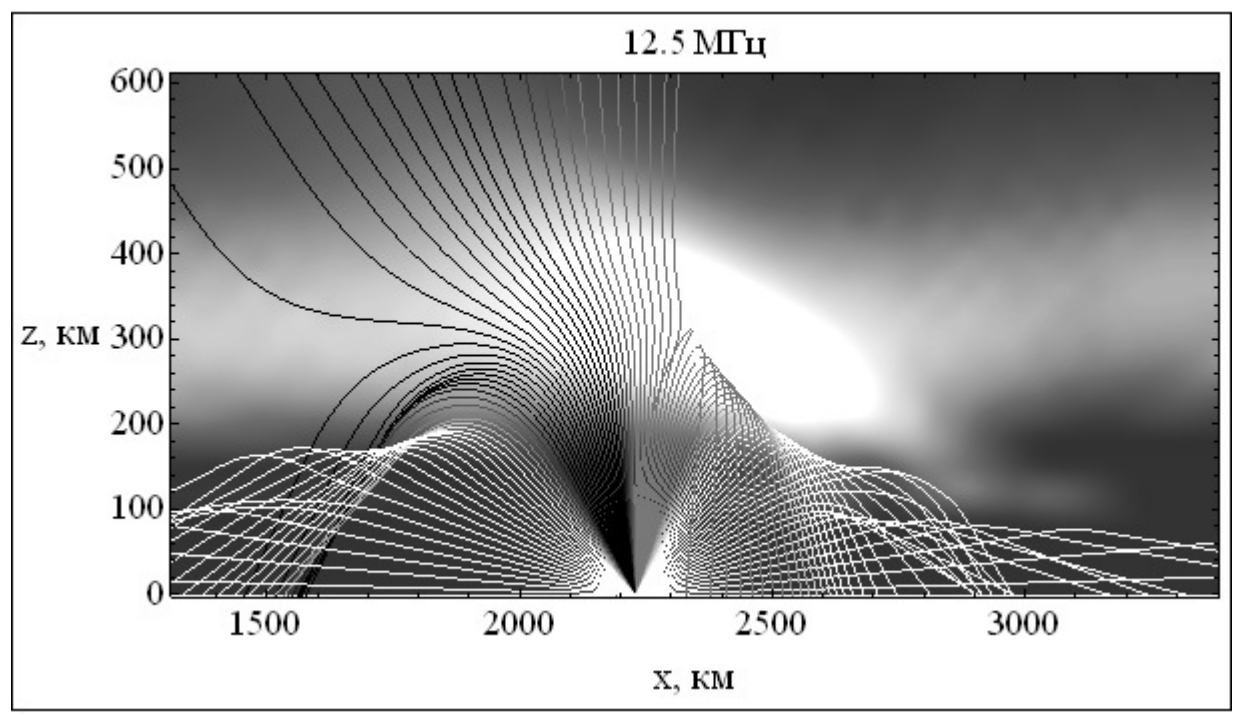

Figure 9: Ray structure. The source of radiation on the Earth, the latitude of $20^{\circ}$ the frequency of $12.5 \mathrm{MHz}$.

Simulation using the profiles of electron density, reduced according to the radio tomography was fulfilled. The models of the ionosphere, taking into account the presence of inhomogeneities as low electron density and high 
electron density were used. Singularities of the propagation of ordinary and extraordinary waves in the ionosphere with the influence of Earth's electromagnetic field are investigated.

In the paper, we researched the propagation of electromagnetic fields at different frequencies in the presence of the equatorial anomaly too. Were considered structure of electromagnetic fields, provided the displacement of the radiation source at altitude and latitude. On the resulting data revealed the influence of the equatorial anomaly in the propagation of decameter signals in the ionosphere. Data on the impossibility of signal propagation in the case of a source of radiation are close to the nucleus equatorial anomaly, were obtained too. The results obtained are approximate, but should help to fully explore the features of the propagation of decameter signals in the ionospheric plasma. The authors developed a dynamic model of the ray propagation [3].

\section{Acknowledgements}

This work was supported by RFBR (grants No. 10-02-01103-a; 09-0700189-a).

The authors thank Professor A.P. Anioutine for valuable advice and comments.

\section{References}

[1] Kryukovsky, A.S.; Lukin, D.S.; Rastyagaev, D.V. (2009) "Investigation of the features of propagation of short radiowaves in an inhomogeneous anisotropic ionosphere", Electromagnetic Waves and Electronic Systems 14(8): 17-26.

[2] Kryukovsky A.S.; Lukin, D.S.; Rastyagaev, D.V. (2010) "Simulation of ray and the caustic structure of electromagnetic fields according by ionospheric tomography in the vicinity of the equatorial anomaly", Electromagnetic Waves and Electronic Systems 15(8): 5-11.

[3] Andreeva, E.S.; Kryukovsky, A.S.; Kunitsyn, V.E.; Lukin, D.S.; Rastyagaev, D.V.; Kiryanova, K.S. (2011) "Simulation of ray and the caustic structure of electromagnetic fields according by ionospheric tomography in the vicinity of the equatorial anomaly", in Propagation of Radiowaves, Collection of Reports XXIII Russian Scientific Conference, Vol 3. (23-26 May 2011) Mari State Technical University, Yoshkar-Ola: 288-291. 
[4] Kryukovsky, A.S.; Kiryanova, K.S. (2011) "Dynamic modeling of radiowave propagation in the vicinity of the equatorial anomaly based on the method of bicharacteristics", Electromagnetic Waves and Electronic Systems 16(8): 21-25.

[5] Lukin, D.S.; Spiridonov, J.G. (1969) "Application of the method of characteristics for the numerical solution of radiowave propagation in inhomogeneous and nonlinear area", Radiotechnics and Electronics 14(9): 1673-1677.

[6] Lukin, D.S.; Palkin, E.A. (1982) Numerical Canonical Method in Problems of Diffraction and Propagation of Electromagnetic Waves in Inhomogeneous Media. Moscow Physical and Technical Institute, Moscow.

[7] Kunitsyn, V.E.; Tereshchenko, E.D. (2003) Ionospheric Tomography. Springer.

[8] Andreeva, E.S.; Franke, S.J.; Kunitsyn, V.E.; Yeh, K.C. (2000) “ Some features of the equatorial anomaly revealed by ionospheric tomography", Geophysical Research Letters 27(16) 2465-2468.

[9] Frankem S.J.; Yehm K.C.; Andreevam E.S.; Kunitsyn, V.E. (2003) "A study of the equatorial anomaly ionosphere using tomographic images", Radio Science 38(1): 1011-1020.

[10] Kunitsyn, V.E.; Andreeva, E.S.; Franke, S.J.; Yeh, K.C. (2003) "Tomographic investigations of temporal variations of the ionospheric electron density and the implied fluxes", Geophysical Research Letters 30(16): 1851-1854. 